\title{
Introduction: A view of neurosurgery's legacy in technology
}

\author{
Charles J. Prestigiacomo, M.D., ${ }^{1}$ and Mark C. Preul, M.D. ${ }^{2}$ \\ ${ }^{1}$ Department of Neurological Surgery and Radiology, Neurological Institute of New Jersey, New Jersey \\ Medical School, University of Medicine and Dentistry, Newark, New Jersey; and ${ }^{2}$ Division of Neurological \\ Surgery, Barrow Neurological Institute, St. Joseph's Hospital and Medical Center, Phoenix, Arizona
}

$\mathrm{S}$ CIENCE is the systematized body of knowledge as an object of study. Technology is the application of science to improve everyday life. Although it is logical to expect that the science precedes the technological advance, history abundantly illustrates that at times the craftsman, with meticulous trial and error and not with the foundation of scientific proof, has developed the ofttimes ingenious technological advance. The treatment of intracranial disease in a way is no different. Although it is considered one of the most technologically dependent and advanced fields in medicine, brain or head surgery and its associated technologies, mainly in the form of instruments, existed in prehistoric ages. Today, neurological surgery enjoys a position at the forefront of both science and technology. Arguably neurosurgery relies on technology and its development more than other surgical specialties. Kinsmen to the neurosurgeon are bioengineers, radiation physicists, neuroscientists, nuclear MR scientists, electrophysicists, optical scientists, anatomists, anthropologists, illustrators, and more. Perhaps because the brain is not so accessible as other organs, we have had to produce means to specially invade its environment that are accurate and limited.

Application of technology to what we recognize as an identifiable neurosurgical specialty-and what engendered the capability to perform relatively exact, targeted surgery-stems from the work of Paul Broca and others involved in craniocerebral topography in the late 19 th century. At that time, cranial procedures were still restricted to those involving lesions that could be visualized on the surface of the head or that had erupted through the scalp. Individuals such as Broca, Reid, Taylor, Zernov, Horsley, and others began developing devices and algorithms that would reproducibly localize eloquent structures that lay beneath. Although crude, these craniocerebral topographic methods still have relevance for modern neurosurgery and lie at the historical foundations of technology application in neurosurgery.

This issue of Neurosurgical Focus echoes these beginnings of organized neurosurgery and serves as a com- pendium of some of the more important technological advances in neurological surgery. By no means comprehensive, this collection of articles presents a broad spectrum of whence neurological surgery came and where it may go.

The reader will find several broad themes in this issue with regard to the advancements of technology in neurosurgery. The first theme of the issue focuses on how technology "fits" into the various schema of anatomy, art, culture, and life. Indeed, much of our understanding of the brain and the surgical approaches depended on the ability to realistically represent the object to others in 2 dimensions. The technological advances in organ preservation and illustration were critically if not exclusively important in the ultimate development of what we do today. It is also clear that certain developments cannot occur if cultural, societal, or other technological influences are not at work. This is clearly evinced in the fact that the birth of the computer allowed the practical application of the then theoretical equations that would make CT a reality.

\section{7}

The second theme of this issue revolves around the development of several "tools of the trade." The penetration of the calvarium in a controlled, relatively safe way has been performed since the days of prehistory. Since then, numerous tools have been adapted, borrowed, modified, and reconstructed to help the neurosurgeon safely and effectively accomplish the surgical procedure. Of the many tools in our armamentarium, this issue will focus on the microscope, its cousin the endoscope, and the laser. As with Broca, Reid, Burckhardt, Zernov, and others, localization and the development of technological innovations to aid in this process became tantamount to the practice of our specialty. Stereotaxy, neuronavigation, and radiosurgery all emanate from this one, fundamental need to perfect our ability to precisely and reproducibly localize specific regions of the brain to the submillimeter level. Although very broad in its reach, the marriage of stereotaxy and image guidance, and the subsequent birth 
of radiosurgery, have changed the face of neurosurgery. A very intriguing aspect of our tools, like the Gigli saw, is that at times we find an innovative application for them. The development of vagal nerve stimulation and the rising evidence that suggests several indications for this device are presented in this issue as well.

The final theme that can be seen throughout this issue is the development of techniques and how instruments were born from them. Spinal instrumentation surgery is well represented in this issue and demonstrates how our improved understanding of the biomechanics of the spine is driving the development of new instruments and thus new ways of treating patients with spinal disease.

These articles emphasize the marriage of science and technology in neurological surgery. Without the fortitude to pursue technological breakthroughs or the science behind the techniques used in neurosurgery, the care of the neurosurgical patient would not be where it is today. Recognizing this delicate interplay and using it to its fullest potential in helping our patients is our privilege and our responsibility. This is how neurosurgery will continue to move ahead. 